\title{
Growth pattern for body weight, height at withers and body length of Kalahari Red goats
}

\begin{abstract}
The objective of this research was to examine the growth pattern for body weight, body length and height at withers of Kalahari Red goats using non-linear models. The body size measurement data were collected from 227 Kalahari Red female goats and fit into Gompertz and Brody growth model. The results revealed that Gompertz growth model had the best goodness of fit to describe the growth of Kalahari Red goats for body weight, body length and height at withers as shown by higher coefficient of determination (97.9, 98.9 and 99.1, respectively). The correlation coefficients between A and $\mathrm{k}$ for body weight, body length and height at withers were negative in both models, implying that goats of larger mature size tended to have a slower growth rate in relation to their mature size. Height at withers-body weight has the highest correlation coefficient (0.96).
\end{abstract}

Keyword: Goats; Kalahari Red; Growth models; Mature size 